\title{
JOHN DEWEY: IDEIAS PARA PENSAR NO ENSINO DE FILOSOFIA E SEUS SENTIDOS
}

\author{
María Belén Bedetti ${ }^{1}$ \\ (D) https://orcid.org/0000-0002-5927-5389 \\ Verónica Soledad Walker ${ }^{2}$
}

Resumo: $\mathrm{O}$ artigo defende a inclusão da filosofia como espaço de ensino e aprendizagem na escola. Começa por reconhecer a necessidade de abordar filosoficamente a questão para seu ensino e reconstruir o sentido em que a entendemos como um passo anterior à sua defesa. Nesse quadro, retoma-se a discussão de um ensino centrado na filosofia ou na tradição filosófica, como um debate clássico sobre a didática da filosofia. Como contribuição central, a concepção de filosofia de John Dewey é investigada, e são analisadas as razões para o estudo dessa disciplina, que apresenta uma superação do debate tradicional entre filosofia e filosofar. $O$ autor reivindica a filosofia como "filosofando", embora assuma o estudo da tradição filosófica como necessário para nos emanciparmos das ideias e preconceitos que incorporamos sem reconhecê-los como tais. Ao mesmo tempo, defende o estudo da tradição como um conjunto de ideias que devem ser colocadas em diálogo com o presente e, portanto, podem ser ferramentas para pensarmos sobre os assuntos atuais. Por fim, suscita a curiosidade do sujeito, o sentimento de admiração pelo universo e seus enigmas, como outro grande motivo para o estudo da filosofia.

Palavras-chave: John Dewey. Filosofia. Ensino de filosofia. Sentidos do ensino de Filosofia.

1 Professora em Filosofia. Mestranda em Educação (UNICEN). Professora adjunta de Filosofia da Educação e assistente de Didática Especial de Filosofia (UNS, Bahía Blanca, Argentina). E-mail: belen.bedetti@uns.edu.ar

2 Doutora em educação, atua como professora adjunta do Departamento de Humanidades e de Economia da Universidade Nacional do Sul (UNS, Argentina) com atividades em pesquisa, assumindo a diretoria de um Projeto Grupal de Investigação sobre formação e trabalho da docência no ensino superior. E-mail: verowalk@hotmail.com 


\section{JOHN DEWEY: IDEAS TO THINK ABOUT THE TEACHING OF PHILOSOPHY AND ITS SENSES}

Abstract: The article defends the inclusion of philosophy as a teaching and learning space in educational institutions. It starts from recognizing the need to philosophically approach the question for its teaching and reconstruct the sense in which we understand it as a previous step to its defense. In this framework, the discussion of a teaching centered on philosophy as a course, or on the philosophical tradition is retaken as a classic debate on the didactics of philosophy. As a central contribution, we investigate John Dewey's conception of philosophy and the reasons for the study of this course, which represents an overcoming of the traditional dabte between Philosophy-philosophize. The author claims philosophy as "philosophizing", although he assumes the study of the philosophical tradition as necessary to emancipate ourselves from ideas and prejudices that we have incorporated without recognizing them as such. At the same time, it defends the study of tradition as a set of ideas that should be put into dialogue with the present and, thus, they are tools for thinking about current affairs. Finally, the article raises the subject's own curiosity, the feeling of wonder at the universe and its enigmas, as another great motivating reason for the study of philosophy.

Keywords: John Dewey. Philosophy. Teaching of philosophy. Senses of the teaching of philosophy.

\section{JOHN DEWEY: IDEAS PARA PENSAR LA ENSEÑANZA DE LA FILOSOFÍA Y SUS SENTIDOS}

Resumen: El artículo defiende la inclusión de la filosofía como espacio de enseñanza y aprendizaje en instituciones educativas. Se parte de reconocer la necesidad de abordar filosóficamente la pregunta por su enseñanza y reconstruir el sentido en que la entendemos como paso previo a su defensa. En este marco se retoma la discusión de una enseñanza centrada en el filosofar o en la tradición filosófica, como debate clásico de la didáctica de la filosofía. Como aporte central, se indaga en la concepción de filosofía de John Dewey y se analizan motivos para el estudio de esta disciplina que presentan una superación al debate tradicional Filosofía-filosofar. El autor reivindica a la filosofía como "filosofar", aunque asume al estudio de la tradición filosófica como necesario para emanciparnos de ideas y prejuicios que hemos incorporado sin reconocerlos como tales. A su vez, defiende el estudio de la tradición como conjunto de ideas que deben ser puestas en diálogo con el presente y, así, resultan herramientas para pensar la actualidad. Finalmente, plantea la curiosidad propia del sujeto, el sentimiento de maravilla ante el universo y sus enigmas, como otro gran motivo implusor del estudio de la filosofía.

Palabras clave: John Dewey. Filosofía. Enseñanza de la filosofía. Sentidos de la enseñanza de la filosofía. 
Introducción

La presencia de la filosofía como espacio autónomo en la educación institucionalizada ha recorrido diferentes derroteros. Son muchas las veces en las que se ha intentado quitarla de los planes de estudio y programas del nivel secundario, que es aquel en el que históricamente ha estado más consolidada su presencia. En ocasiones dicha voluntad ha sido acompañada por la acción, y durante períodos ha sido relevada de la formación institucionalizada, quedando fuera de la formación de los jóvenes estudiantes del nivel medio. Es que hay cierta idea acerca de la "inutilidad" de la filosofía que la vuelve blanco de distintos ataques en el marco de sistemas educativos que privilegian, como ya anticipara Martha Nussbaum (2010) en Sin fines de lucro, una formación de corte tecnicista, que apunta a la adquisición de habilidades y saberes ponderados en el mercado global.

En la actualidad existen distintos movimientos y agrupaciones en países de Latinoamérica -aunque también en Europa- en los que se busca generar resistencias ante las posiciones que pretenden quitar a la filosofía de la escuela secundaria. Es en este sentido que hoy se vuelve necesario renovar el esfuerzo por pensar y generar argumentos en torno a los sentidos de su enseñanza en las escuelas. Recurrir a la tradición y a los/as autores de la misma, puede proveernos de herramientas de reflexión para dicha tarea.

En este trabajo, y como respuesta a esta coyuntura histórica, social y política latinoamericana, en la que la filosofía pelea por un lugar en la formación de los jóvenes, se indagará, particularmente, en las ideas del pensador norteaméricano John Dewey en torno a la filosofía y su enseñanza.

Para ello, y a partir de reconocer que no existe una única manera de entender a esta disciplina y, en consecuencia, tampoco una única forma de enseñarla, se sostendrá que el problema de la enseñanza de la filosofía es en sí mismo un problema filosófico (CERLETTI, 2008). Esto se debe a que cada vez que surge la pregunta por su enseñanza -en docentes, funcionarios públicos encargados de las políticas educativas, didáctas de la filosofía y filósofos de la educación- es necesario un planteo de qué se entiende como filosofía, planteo que está a la base de cualquier posterior enseñanza, de cualquier inclusión o exclusión de los diseños curriculares y programas escolares. Esto es, antes de 
definir el cómo enseñar filosofía, es necesario dar cierta discusión acerca de qué se enseña en nombre de la misma; antes de considerar su valor en la enseñanza del nivel secundario, cabe preguntarnos a qué nos referimos cuando hablamos de filosofía.

Por ello, pensar y asumir una respuesta personal y/o colectiva ante este interrogante, nos permite también reconocer que quizás no sea cualquier forma de filosofía la que queremos defender como necesaria en la enseñanza institucionalizada ni en los niveles obligatorios de la misma. Y es aquí donde el problema se vuelve filosóficodidáctico, pues nos interpela a buscar razones, matices, conceptos.

En este trabajo se partirá del rastreo de la concepción de filosofía que sostiene John Dewey para, posteriormente abrevar en su trabajo acerca de la reconstrucción de esta disciplina que propone para la filosofía de postguerra. Finalmente, se buscará identificar su postura en torno a la importancia del estudio de la filosofía, mientras que se indaga en cuál es la filosofía que sostiene como relevante para la formación del sujeto contemporáneo, dando cuenta de sus argumentos y reflexiones. Para esta última tarea se profundizará en la propia voz del autor en su texto titulado Why Study Philosophy? (1971), orientada por el análisis sobre este problema que se plasma en el texto Dewey e a Filosofia, de autoría de Leoni Maria Padilha Henning (2019).

En definitiva, se busca partir de un análisis filosófico sobre el problema de qué es la filosofía y su sentido en la formación del sujeto. Esto permitirá fundamentar el estudio de la filosofía como disciplina del conocimiento y como forma del pensar, lo que a su vez servirá como puntapié para pensar una didáctica específica de la filosofía que oriente la tarea docente.

\section{John Dewey y su concepción de la filosofía}

En Argentina, muchos de los más reconocidos autores e investigadores de la didáctica de la filosofía, sostienen la cualidad filosófica de la pregunta por la enseñanza de esta disciplina; a partir de esto afirman que su enseñanza es un problema filosófico - 
argumentos en torno a ello podemos leer en Obiols (2008), Cerletti, (2008) y Ranovsky, (2011), entre otros-.

Esta idea se distancia de aquella mirada más tradicional que concibe a la didáctica como un campo únicamente instrumental del saber; de esta manera se afirma, en contrapartida, la necesidad de una didáctica filosófica que antes y durante la reflexión acerca del cómo enseñar, problematice qué se enseña y el mismo sentido de la enseñanza de esta disciplina. Estas cuestiones se constituyen, entonces, en un problema filosófico y, de esta manera, han planteado que es necesaria la tarea de encarar una filosofía de la enseñanza de la filosofía. Así,

Convertir la cuestión de 'enseñar filosofía' en un problema filosófico modifica también la secuencia tradicional de la didáctica de la filosofía, que privilegia el 'cómo' enseñar, para poner en primer lugar el análisis del 'qué' enseñar. El 'qué' [...] involucra una toma de posición frente a la filosofía y al filosofar (CERLETTI, 2008, p. 60).

Si se pretende defender la presencia de la filosofía en las escuelas es necesario acompañar tal posicionamiento por una caracterización de la filosofía en la que se está pensando, pues, en tanto problema filosófico, el qué sea la filosofía no encuentra respuestas ni definiciones absolutas. De esta manera, muy diversos serán los argumentos que pueden sostenerse si se entiende a la filosofía como conjunto de habilidades para el pensar, como compendio de ideas y conceptos de la tradición, como modo de vida, como herramienta para la construcción de conceptos, como legado cultural de nuestra historia, entre otras tantas posibilidades de respuestas. Es posible rastrear, en la historia de la filosofía, una gran cantidad de concepciones que se diferencian entre sí y entran en discusión, acerca de a qué nos referimos cuando hablamos de filosofía.

Entre estos filósofos y tradiciones podemos encontrar a John Dewey, quien afirma que la filosofía no puede entenderse como un saber verbalista ni meramente especulativo, sino que tiene un anclaje directo en la experiencia de los sujetos. Para él, y en firme discusión con concepciones radicalmente diferentes, afianzadas en ese momento histórico y que abrevaban en tradiciones anteriores,

Siempre que se ha tomado en serio la filosofía se ha supuesto que significa la adquisición de una sabiduría que influiría en la conducta de la 
vida. Lo demuestra el hecho de que casi todas las antiguas escuelas de filosofía se organizaron también como modos de vida, hallándose obligados los que aceptaban sus principios a ciertos modos concretos de conducta [...] (DEWEY, 2004b, p. 272).

A diferencia de las ciencias, que delimitan sus objetos de estudio y los saberes particulares que producen, la filosofía se constituye en la reflexión y adopción de cierta disposición general frente a la realidad, frente al mundo. Así, aspira a la totalidad y busca integrar todos los diferentes aspectos de la experiencia que en ocasiones entran en conflicto. Es por ello que Dewey asume un vínculo casi de identidad entre filosofía y teoría de la educación, pues concibe a la educación como el proceso mediante el cual los individuos adquieren ciertas disposiciones generales ante la naturaleza y los seres humanos.

Si se sigue el análisis de Philip Jackson (2004) en John Dewey y la tarea del filósofo, hay tres características que pueden detectarse a la base de la concepción de la filosofía del autor norteamericano, y que nombra como "los tres compromisos filosóficos de Dewey" (JACKSON, 2004, p. 112 y ss.): la primera es que esta es una empresa empírica, y ha de rechazarse la ambición de trascender la experiencia; la segunda es que busca ser valiosa (también podríamos decir "útil") para mejorar a la sociedad; la tercera es que se constituye en una actividad intelectual, que utiliza ideas abstractas pero ha de estar estrechamente vinculada a los asuntos de la vida cotidiana .

Se asume así a la filosofía como anclada en la experiencia cotidiana, llamada en ocasiones con el nombre de "naturalismo empírico", en tanto se presenta en clara oposición de aquellas filosofías que se alejan del mundo real en busca de una verdad inmutable, y que se constituyen en saberes abstractos, más bien asociados a la metafísica. Esta filosofía no es una práctica desinteresada, como muchos han concebido tradicionalmente, es una búsqueda del saber que tiene un sentido definido, concreto: el de mejorar nuestra propia experiencia, nuestra realidad cotidiana. Es por ello que debe partir de la vida del sujeto que la realiza y de los problemas que surgen desde la cotidianeidad.

En definitiva, y a riesgo de simplificar demasiado, se puede decir que en Dewey la filosofía se constituye en una forma de saber en estrecha relación con la experiencia del 
ser humano, que parte de sus intereses cotidianos y que tiene la pretensión de generar transformaciones entendidas como perfeccionamientos o mejoras a la vida diaria. Si se quisiera defender su enseñanza, desde esta perspectiva, habría que apuntar a estas características tanto para dar cuenta de por qué ha de ser importante su inclusión en la escuela como para pensar el cómo ha de llevarse a las aulas en tanto saber a enseñar y aprender.

\section{La reconstrucción de la filosofía como tarea contemporánea}

La concepción de filosofía presentada puede desprenderse y reconstruirse desde la mirada crítica que el autor plantea en trono a las filosofías anteriores y contemporáneas a su pensamiento. En este sentido, La reconstrucción de la filosofía resulta un texto sumamente valioso en tanto analiza el estado actual de la filosofía a partir de su desarrollo histórico. En este escrito Dewey rastrea, en la historia de esta disciplina, los motivos que han llevado a que tome esta forma particular, mientras que también señala y analiza los motivos por los que tal filosofía no responde al nuevo contexto de posguerra. Además, el autor la proyecta hacia un futuro en tanto propone sentidos de su reconstrucción.

El texto está compuesto por una serie de conferencias que tuvieron lugar luego de la primera guerra mundial. Posteriormente, y luego de la revisión del autor veinticinco años más tarde, se reedita la obra y se reafirma el posicionamiento original, manteniendo el texto sin modificaciones. No obstante, esta reedición es presentada con una nueva introducción en la que, entre otras cuestiones, Dewey responde a algunas de las críticas recibidas en torno a la publicación original.

Sin ánimo de ser exhaustivo, en este apartado se presentan algunas de las ideas centrales que manifiesta el autor en este texto y que podrían considerarse una especie de manifiesto pragmatista, ya que en él, el mismo Dewey no solo asume posición, sino que arma la "agenda" de la filosofía del futuro.

En relación con la filosofía de su época, el autor presenta su origen y desde allí reconstruye algunos de sus aspectos fundamentales: sostiene que su tarea, en sus primeros tiempos, fue la de justificar racionalmente las creencias y costumbres 
tradicionales. A raíz de la imposibilidad de ser aceptadas por mera costumbre o por la autoridad social, ciertos saberes requirieron una justificación basada en demostraciones lógicas rigurosas; es por ello que afirma, como cuestionamiento a la filosofía que aun se profesa, que "Esta actitud, llevada a su extremo peor, ha reducido la filosofía a una exhibición de terminología complicada, de lógica sutilizadora, de falsa devoción a las formas simplemente externas de la demostración completa y minuciosa" (DEWEY, 1964, p. 86). Esta marca de origen ha acompañado a las filosofías clásicas en su presunción y en la pretensión de ser definitivas.

En este deseo por poder dar razón de la realidad última, la filosofía ha separado dos reinos: el de la experiencia y el de la realidad trascendente, absoluta, abocándose a ésta última y olvidando su origen primitivo en el deseo y el drama de la existencia cotidiana del hombre. En general las filosofías clásicas, a pesar de que pueden encontrarse diferencias significativas entre ellas, comparten el partir de este supuesto de la realidad escindida, y de una mirada que da primacía a lo trascendente por sobre lo inmanente.

Es en la figura de Francis Bacon que Dewey encuentra el modelo de filósofo que comienza con la tarea de revisar este supuesto tan arraigado a lo largo de la historia de la disciplina. Es por ello por lo que lo asume, por llamarlo así, como "profeta" del pragmatismo: en virtud de su crítica al conocimiento antiguo y medieval, al cuestionamiento de los métodos demostrativos, el principio de autoridad y el silogismo aristotélico tan presentes en la historia de la filosofía. La investigación será para Bacon la única manera de conocer a la naturaleza. A su vez, el avance continuo en el conocimiento a través de la experiencia, permitiría que no quedaran arraigados ciertos saberes por mucho tiempo, a modo de dogmas que luego resultan difíciles de cuestionar y de identificar.

No obstante, la reconstrucción implica un proceso que no es dable de un momento para el otro,

No es cosa fácil para los hombres el desprenderse de sus viejos hábitos de pensar, y jamás pueden echarlos de sí todos juntos de una sola vez. Al desarrollar, señalar y recibir ideas nuevas nos encontramos en la necesidad de servirnos de alguna de las antiguas como instrumentos de entendimiento y de comunicación (DEWEY, 1964, p. 141). 
A lo antes mencionado también se suman las ideas de verdad y falsedad, taxativas, con las que se busca clasificarlo todo, al igual que con la realidad suprema y la imperfecta, con la razón y la experiencia. En particular la idea de verdad, pensada desde un sentido pragmatista, rechaza esta oposición de ideas sosteniendo la conciliación entre los polos; polos que los dualismos -o como él los llama en varias de sus obras, "las filosofías de lo uno o lo otro" - han instalado como irreconciliables. Por ello el filósofo cierra el texto afirmando que

Cuando la filosofía haya cooperado con el curso de los acontecimientos y haya hecho claro y coherente el significado del detalle diario, la ciencia y la emoción se compenetrarán mutuamente; la práctica y la imaginación se darán un abrazo. La poesía y el sentido religioso brotarán entonces como flores espontáneas de la vida. La tarea y el problema de la filosofía durante la época de transición consistirán en favorecer esa articulación del curso corriente de los hechos y de la revelación de sus significados (DEWEY, 1964, p. 276).

\section{La enseñanza de la filosofía}

El debate canónico en torno a la enseñanza de la filosofía suele enmarcarse en dos posturas que se han entendido como antagónicas y que son representadas por figuras de filósofos tan reconocidos como Hegel y Kant. En el caso del primero, se hace referencia a esta cuestiónen en algunos fragmentos de sus textos como la Fenomenología del espíritu y los Escritos pedagógicos. En estos afirma que la filosofía es su historia y no hay manera de aprenderla sin un abordaje de su tradición. Por su parte, Kant $(1943 ; 1973)$ afirma que no hay algo definido y cerrado a lo que se pueda llamar "Filosofía", y que por tanto solo es posible enseñar a filosofar. Estas reflexiones aparecen específicamente tanto en la Crítica de la razón pura como en Sobre el saber filosófico.

Como puede verse, la tensión que se presenta en este debate está puesta en entender a la filosofía como un sustantivo (como una disciplina específica) o como un verbo (como una acción, una práctica). Y claramente muy diferente será la forma de enseñanza que se escoja si se posiciona en uno u otro extremo de la discusión. Si se entiende de esta manera el debate no es difícil sospechar que Dewey se opondría a los 
términos del mismo por su crítica a las "filosofías de lo uno o lo otro" o a aquellas posturas que emergen como negación de otra visión. Ahora bien, en este caso se ha señalado (OBIOLS, 2008) que esta es en realidad una falsa dicotomía pues no necesariamente los autores han negado lo que se entiende como perspectiva opuesta, sino que han hecho hincapié en otra característica de la filosofía. Lo que esto quiere decir es que Hegel no afirma que la enseñanza de la filosofía no implique aprender a filosofar, sino que hacerlo involucra conocer el filosofar de los filósofos de la tradición. Por el otro lado, en el caso de Kant, no afirma que la historia de la filosofía no deba ser enseñada o aprendida, si no que en todo caso su abordaje debe realizarse con la finalidad específica de aprender a filosofar, pues la Filosofía no es un campo del saber cerrado, desarrollado plenamente.

Quizás el énfasis en una u otra cara puede depender de la circunstancia histórica que nos toque vivir. $\mathrm{Si}$, en un cierto momento, reaccionamos contra el academicismo que en nombre de la filosofía, de la rigurosidad del contenido y de los textos 'sagrados' inhibe la expresión del pensamiento propio, probablemente podamos aferrarnos y defender, con justicia en las circunstancias señaladas, la propuesta de aprender a filosofar. Si, por el contrario, en otro marco, por imperio de la pedagogía formalista o de la simple demagogia, nos sentimos hartos de que en nombre del aprender a filosofar, éste se vacíe de contenido filosófico, es probable que nuestra reacción adquiera un sentido contrario al anterior (OBIOLS, 2008, p. 65).

En el caso de Dewey se puede ver que existe una postura que media entre estas dos formas extremas de pensar la enseñanza de la filosofía, pues reconoce el valor de su tradición -y de su conocimiento-, a la vez que promueve una enseñanza para la acción, para el filosofar. En Why Study Philosophy? Dewey (1971) afirma que un motivo para estudiar filosofía

[...] es que existieron filósofos antes que nosotros y el único camino para evitar ser dominado por ellos es volvernos filósofos nosotros mismos. Si es necesario un ladrón para atrapar a otro ladrón, es simple sospechar que se necesita a un filósofo para atrapar a otro filósofo (DEWEY, 1971, p. 62, traducción de la autora). 
La dominación de la que habla Dewey apunta a ciertos supuestos, prejuicios, valores e ideas que, instalados por la tradición, constituyen las lentes desde las cuales se percibe la realidad y, si no pueden ser reconocidas como tales, no permitirán verla de otras maneras. Es así como

\begin{abstract}
Mientras mueren como filosofía, las ideas viven en la vida intelectual corriente e inconsciente del hombre en general. Ellas se vuelven los presupuestos a la base, las premisas no expresadas, las herramientas del pensamiento y la acción (y por lo tanto quienes lo controlan). Infiltradas a través de medios como la educación, la ley, el lenguaje, la religión y la ciencia misma, ellas se adueñan de nosotros. A menos que seamos dominados por ellas, nosotros debemos dominarlas. Y esto implica un continuo esfuerzo por sacarlas afuera de nuestros lugares inconscientes escondidos; una revisión deliberada y reflexiva de ellas - lo que es decir, el estudio de la filosofía (DEWEY, 1971, p. 63, traducción de la autora).
\end{abstract}

Un buen motivo para aprender filosofía -y para enseñarla- es, entonces, el emanciparnos de sus ideas a partir de conocerlas e identificar cómo operan en nuestra percepción del mundo. Para ello habrá que buscar en la tradición filosófica, en su historia, cómo se han construido tales sistemas que hoy se encuentran incorporados a nuestra forma de ver el mundo. Este ha sido el trabajo de la filosofía crítica, a la que Dewey compara con la realización de un inventario: es la tarea de contar e identificar aquellas ideas que operan en nuestro "depósito de ideas", para poder recurrir a ellas cuando se quiera, pero también para reconocer su origen histórico. De esta forma, la tarea de la crítica es la de realizar

[...] un inventario intelectual que nos va a decir dónde estamos parados, cuáles son nuestros recursos y cuáles nuestras deudas; un inventario que tiene por objetivo permitirnos manejar nuestro pasado, en lugar de ser manejado por él; el que podamos caminar hacia adelante más libre y valientemente, menos obstaculizados por las sombras que vienen del pasado (DEWEY, 1971, p. 64, traducción de la autora).

Ahora, a pesar de la gran importancia que Dewey otorga al estudiar y reconocer en el pasado de la filosofía aquellas ideas, métodos y disposiciones que nos afectan actualmente, esta no es la única finalidad con la que puede enseñarse o aprenderse la filosofía: 
Está implícito en lo que se ha dicho que la filosofía, mientras nos deja entrar en el secreto de las ideas y conductas que fueron creadas en el pasado, también construye, $\mathrm{o}$ al menos reconstruye, herramientas intelectuales para su uso presente. En la medida en que esto es así nos encontramos con otro motivo para estudiar filosofía (DEWEY, 1971, p. 64-65, traducción de la autora).

Estudiar filosofía es también una forma de aprender a filosofar en nuestro tiempo, desde nuestro horizonte de sentido (GADAMER, 1996) entablar un diálogo con la tradición. Así, la filosofía no ha de ser entendida como una compilación de tradiciones y nombres propios sino como una disciplina en la que se pone en juego el filosofar, pues, como afirma Sanchez Meca, no tiene sentido plantear la historia de la filosofía como galería de filósofos o de sus sistemas con un objetivo exclusivamente de coleccionista, sin pensar sus relaciones con el presente. En sus palabras:

Nada de extraño tiene entonces plantear la historia de la filosofía como historia de la recepción de las obras filosóficas, de sus interpretaciones y reinterpretaciones hasta llegar a nosotros, de tal forma que se ponga de manifiesto como cada obra actuando sobre el horizonte de pensamiento de una determinada época, le afecta y contribuye a su transformación (SANCHEZ MECA, 1996, p. 313).

Entonces, es necesaria la filosofía en tanto estudio del pasado y de las ideas que constituyen nuestra manera de entender la realidad, los "filtros" desde los cuales se percibe e interpreta el mundo, y las herramientas con las que se cuenta para hacerlo; pero no solo eso, también es necesaria en tanto permite pensar el presente, posición desde la que se acentúa la perspectiva del filosofar en la actualidad.

Ahora bien, Dewey, plantea una última razón para el estudio de la filosofía, una razón que es intrínseca a los sujetos:

El último 'porqué' para estudiar filosofía es que hay quienes que no necesitan ningún 'porqué', los que la estudian por inclinación [...]. Siempre que se encuentren una mente curiosa y un universo maravilloso, siempre quedará la mejor de las razones para estudiar filosofía: la necesidad (DEWEY, 1971, p. 65, traducción de la autora). 
De acuerdo con esto, podemos pensar si hoy, los estudiantes de la escuela secundaria a quienes se dirige la enseñanza de la filosofía, viven la "necesidad de saber" que genera este asombro ante el universo. En ocasiones se da por supuesto que lo que a los adultos nos conmueve, nos invita a pensar, también hará lo suyo con los jóvenes. No obstante, para Dewey

Nosotros violentamos la naturaleza del niño y dificultamos los mejores resultados éticos al introducir al niño demasiado rápidamente en una cantidad de estudios especiales, de lecturas, escritura, geografía, etc. sin relación con su vida social. El verdadero centro de correlación de las materias escolares no es la ciencia, ni la literatura, ni la historia, ni la geografía, sino las propias actividades sociales del niño (DEWEY, 1967, p. 58).

En función de este último motivo, y desde nuestro rol de docentes de filosofía, será necesario reconocer y estimular en el otro esa mirada del asombro, de indagación permanente, para poder invitarlo a ver al universo como algo maravilloso. Esta tarea no siempre es fácil en nuestras escuelas secundarias actuales, sobre todo en el marco de la sociedad contemporánea, a la que se le ha diagnosticado la pérdida de la capacidad de tener experiencias anunciada por Walter Benjamin (1989) en Experiencia y pobreza. Poder salir de esa mirada naturalizadora, rutinaria y automática es el camino que posibilitará el hacer palpable la necesidad de la filosofía en el otro, así como estar abierto ante las diferencias que puedan surgir entre sus intereses, realidades y concepciones. Tener experiencias de este tipo en el marco de la enseñanza institucionalizada de la filosofía es un desafío que se puede asumir pensando en el principio de la continuidad de la experiencia que postula Dewey, para quien una buena experiencia es aquella que amplíe la posibilidad y el deseo de tener más experiencias:

Todo depende de la cualidad de la experiencia que se posee. La cualidad de cualquier experiencia tiene dos aspectos. Hay un aspecto inmediato de agrado o desagrado, y hay su influencia sobre las experiencias ulteriores. Lo primero es evidente y fácil de juzgar. El efecto de una experiencia no se limita a su apariencia. Ello plantea un problema al educador. La misión de éste es preparar aquel género de experiencias que, no repeliendo al alumno, sino más bien incitando su actividad, sean sin embargo más que agradables inmediatamente y provoquen experiencias futuras deseables [...] el problema central de una educación basada en la experiencia es seleccionar aquel género de experiencias 
presentes que vivan fructífera y creadoramente en las experiencias subsiguientes (DEWEY, 2004a, p. 73).

La escuela debe ser un espacio para la experiencia filosófica -como aprendizaje de su historia, como filosofar sobre nuestro presente y como necesidad ante lo asombroso del universo- teniendo en cuenta que la buena experiencia filosófica traerá encadenadas otra cantidad de reflexiones y experiencias.

\section{Conclusión}

A lo largo del trabajo pudimos identificar el problema de la fundamentación de la enseñanza de la filosofía como problema que parte de la necesidad de definir, en primer lugar, qué es lo que entendemos bajo el nombre de esta disciplina. A partir de allí se rastrearon, en distintos textos de John Dewey, aportes para construir su propia concepción de la misma, ampliados desde su perspectiva crítica frente a las filosofías clásicas y sus supuestos dualistas, que priorizaron una lectura metafísica de la realidad. Desde esta concepción se reconstruyó el posicionamiento del autor en torno a la enseñanza de la filosofía y sus sentidos, poniéndolo en diálogo con debates caónicos en el marco de la enseñanza de la filosofía.

Desde allí encontramos el valor que se le otorga, desde la perspectiva del autor, al filosofar como actividad práctica. Esto en el marco de ideas que no desacreditan el estudio de la tadición filosófica como ideas que nos anteceden, y que por el contrario lo reivindican ya que, si las desconocemos, pueden dominarnos e imposibiitarnos la construcción de una nueva lectura de la realidad. A su vez, las ideas y conceptos construidos en la historia de la filosofía, para el filósofo norteamericano, pueden ser puestas en diálogo con nuestro presente, constituyéndose en herramientas y valiosos recursos para pensar la actualidad. Por último, pero muy relevante a su vez, el autor señala que uno de los sentidos de la filosofía, su enseñanza y aprendizaje, es acompañar y despertar la curiosidad y el deseo de saber del ser humano, como condición propia. Por 
estos tres distintos motivos perfectamente integrados a sus concepciones generales, Dewey defiende el estudio de la filosofía.

Estas discusiones y tensiones acerca de qué enseñamos en nombre de la filosofía, nos invitan a su vez a pensar lo que sucede en otras disciplinas de las ciencias sociales y humanas. En ellas también puede aparecer la distinción entre una enseñanza centrada en la tradición histórica, el pensar disciplinar como actividad o distintas formas y grados de abordaje de ambas de manera conjunta.

Pudimos reconocer, a lo largo de este trabajo, que la distancia en el tiempo que media nuestra actualidad a la del autor no hace mella en la vigencia de su pensamiento, toda vez que seguimos cuestionando a una filosofía abstracta, demasiado preocupada por la tarea del pensar, que escinde a la realidad y al mismo sujeto, que defiende y se enorgullece muchas veces de su "inutilidad" práctica. Por ello, sus reflexiones nos invitan a seguir reconstruyendo a la filosofía desde las aulas, atendiendo a la experiencia de los sujetos como fuente del filosofar, a la desnaturalización de los supuestos como forma de emancipación de la mirada, y a la acción y la transformación como consecuencia deseable de la misma.

\section{Referencias}

BENJAMIN, W. Experiencia y pobreza. En: BENJAMIN, W. Discursos interrumpidos I. Madrid: Taurus, 1989. p. 165- 173.

CERLETTI, A. La enseñanza de la filosofía como problema filosófico. Buenos Aires: Del Zorzal, 2008.

DEWEY, J. La reconstrucción de la filosofía. Buenos Aires: Aguilar, 1964.

DEWEY, J. El niño y el programa escolar: mi credo pedagógico. Buenos Aires: Losada, 1967.

DEWEY, J. Why study philosophy? En: DEWEY, J. The early works, 1882-1898. London: Carbondale and Edwardsville; Amsterdam: Souththern Illinois University Press: Feffer \& Simmons, 1971. v. 4, p. 62-65

DEWEY, J. Experiencia y educación. Madrid: Biblioteca Nueva, 2004a.

DEWEY, J. Democracia y educación. Madrid: Morata, 2004b. 
GADAMER, H. G. Verdad y método II. Salamanca: Ediciones Sígueme, 1996.

JACKSON, P. John Dewey y la tarea del filósofo. Buenos Aires: Amorrortu, 2004.

KANT, I. Sobre el saber filosófico. Madrid: Adán, 1943.

KANT, I. Crítica de la razón pura. Buenos Aires: Losada, 1973.

NUSSBAUM, M. Sin fines de lucro: por qué la democracia necesita de las humanidades. Buenos Aires: Katz Editores, 2010.

OBIOLS, G. Una introducción a la enseñanza de la filosofía. Buenos Aires: Del Zorzal, 2008.

HENNING, L. M. P. Dewey e a filosofia. En: VELASCO, P. del N. (org.). Ensino de? qual? filosofia: ensaios a contrapelo. Marilia: Editora UNESP, 2019. p. 205-224.

RANOVSKY, A. Filosofía del docente filósofo. Buenos Aires: Colisión, 2011.

SANCHEZ MECA, D. La historia de la filosofía como hermenéutica. Madrid: Universidad Nacional de Educación a Distancia, 1996. 